\title{
A Desmoplakin Point Mutation with Enhanced Keratin Association Ameliorates Pemphigus Vulgaris Autoantibody-Mediated Loss of Cell Cohesion
}

\author{
Carina Dehner, Vera Rötzer, Jens Waschke, and Volker Spindler
}

From the Institute of Anatomy and Cell Biology, Ludwig-Maximilians-Universität, Munich, Germany

\author{
Accepted for publication \\ May 22, 2014. \\ Address correspondence to \\ Volker Spindler, M.D., or Jens \\ Waschke, M.D., Institute of \\ Anatomy and Cell Biology, \\ Department I, Ludwig- \\ Maximilians-Universität, \\ Pettenkoferstraße 11, 80336 \\ München, Germany. E-mail: \\ volker.spindler@med.uni- \\ muenchen.de or jens.waschke@ \\ med.uni-muenchen.de.
}

\begin{abstract}
Desmoplakin (DP) serves to anchor intermediate filaments in desmosomal complexes. Recent data suggest that a specific DP point mutation (S2849G) exhibits increased keratin filament association and fosters $\mathrm{Ca}^{2+}$ insensitivity of desmosomes in keratinocytes, presumably by rendering DP inaccessible for protein kinase $\mathrm{C}(\mathrm{PKC})$ phosphorylation. Previously, we have reported that depletion of the desmosomal adhesion molecule desmoglein (Dsg) 3 induced by autoantibodies from patients with the blistering skin disease pemphigus vulgaris (PV) IgG is reduced in maturated desmosomes and dependent on PKC signaling. We investigated the role of DP-S2849G for loss of cell cohesion mediated by PV-IgG. In cell dissociation assays, expression of green fluorescent protein-tagged DP-S2849G (DP-S2849G-GFP) increased cell cohesion in two different human keratinocyte cell lines and ameliorated loss of cell adhesion induced by pemphigus autoantibodies. Depletion of Dsg3 was inhibited by DP-S2849G-GFP in the cytoskeletal (Triton X-100 insoluble) fraction, and keratin filament retraction, a hallmark of PV, was efficiently blocked similar to treatment with the PKC inhibitor Bim-X. We found that DP is phosphorylated after incubation with PV-IgG in a PKC-dependent manner and that DP-S2849G-GFP expression prevents DP phosphorylation and increases association of PKC- $\alpha$ with PKC scaffold receptor for activated C-kinase 1. Taken together, our data indicate that DP phosphorylation at S2849 represents an important mechanism in pemphigus pathogenesis, which, by reversing $\mathrm{Ca}^{2+}$ insensitivity, promotes Dsg3 depletion. (Am J Pathol 2014, 184: 2528-2536; http://dx.doi.org/10.1016/j.ajpath.2014.05.016)
\end{abstract}

Desmoplakin (DP) is part of the desmosome, a specialized structure necessary for strong intercellular cohesion. ${ }^{1}$ Desmosomes are found in different tissues that are exposed to mechanical stress (ie, cardiac muscle and the epidermis). ${ }^{2,3}$ The desmosomal core is composed of desmogleins (Dsgs) 1 through 4 and desmocollins (Dscs) 1 through 3, transmembrane glycoproteins of the cadherin family. They bind to their counterparts on the adjacent cell with their extracellular domains in a $\mathrm{Ca}^{2+}$-dependent manner, whereas the cytoplasmic tails communicate with linker proteins of the armadillo and the plakin families. DP interacts with plakoglobin, an armadillo family protein that is directly connected to Dsgs and Dscs, as well as to another subgroup of the armadillo family, the plakophilins. Keratin filaments as part of the cytoskeletal network are attached to the adhesion complex via $\mathrm{DP}^{2} \mathrm{Be}-$ sides its function as an adaptor molecule, DP was also found to contribute to signaling pathways, such as the Wnt/ß-catenin signaling pathway. ${ }^{4}$ Furthermore, DP mutations are known to cause a variety of human diseases, such as Carvajal syndrome, which comprises severe cardiomyopathy, woolly hair and keratoderma, ${ }^{5}$ or lethal acantholytic epidermolysis bullosa. ${ }^{6}$

Recently, DP was reported to regulate the maturation of desmosomes to establish a so-called hyperadhesive state. Under these conditions, desmosomes are still adhesive despite the lack of extracellular $\mathrm{Ca}^{2+} .8$ A specific DP point mutation [green fluorescent protein-tagged DP point mutation S2849G (DP-S2849G-GFP)], in which the serine residue is replaced by glycine, exhibits increased anchorage of keratin filaments and fosters $\mathrm{Ca}^{2+}$ independency. The mutation is located in a protein kinase $\mathrm{C}(\mathrm{PKC})$ consensus

Supported by Deutsche Forschungsgemeinschaft, Germany, grant SP1300-1/1 (V.S.) and the Förderprogramm Forschung und Lehre of the Ludwig-Maximilians-Universität (grant 7/11).

Disclosures: None declared. 
sequence, which is consistent with the observation that PKC signaling prevents hyperadhesion. ${ }^{9,10}$ Thus, although this was never tested, it is possible that hyperadhesion may be regulated by PKC-dependent DP phosphorylation.

Desmosomal maturation was also implicated in the autoimmune blistering skin disease pemphigus vulgaris $(\mathrm{PV}),{ }^{11}$ in which Dsg1 and Dsg3 are targets of pathogenic autoantibodies. Patients develop blisters within the oral mucosa and/or the epidermis. A central mechanism after autoantibody binding is the internalization of Dsg3 and Dsg1. ${ }^{12-15}$ Previously, cultured human keratinocytes with immature desmosomes were reported to be more prone to depletion of Dsg3 induced by PV-IgG compared with hyperadhesive cells. ${ }^{16,17}$ It is known that PV-IgG induces activation of $\mathrm{PKC},{ }^{18}$ and the depletion of Dsg3 was prevented by inhibition of PKC. ${ }^{16}$ Because the pathogenic effects of autoantibodies in PV may at least in part depend on $\mathrm{Ca}^{2+}$ sensitivity, we investigated the effect of DP-S2849GGFP on loss of cell cohesion and Dsg3 depletion in PV.

\section{Materials and Methods}

\section{Cell Culture and Plasmid Transfection}

The human immortalized keratinocyte cell line HaCaT and the inducible epithelial A431 cell line were cultured in Dulbecco's modified Eagle's medium that contained 1.8 $\mathrm{mmol} / \mathrm{L} \mathrm{Ca}{ }^{2+}$ supplemented with $10 \%$ fetal calf serum (Biochrom, Berlin, Germany), $50 \mathrm{U} / \mathrm{mL}$ of penicillin $\mathrm{G}$, and $50 \mu \mathrm{g}$ of streptomycin. Cells were kept in a humidified atmosphere by $5 \% \mathrm{CO}_{2}$ at $37^{\circ} \mathrm{C}$. For plasmid transfection, $\mathrm{HaCaT}$ cells were grown to $80 \%$ confluence before transfection with DP-S2849G-GFP, DP-GFP, or an empty GFP vector (1.5 $\mu \mathrm{g}$ of DNA) as controls using Lipofectamine LTX (Life Technologies, Carlsbad, CA) according to the supplier's protocol. Best expression of the constructs was found 48 hours after transfection. For induction of the DPS2849G-GFP point mutation, A431 cells were kept at least 24 hours in medium that contained doxycycline (Santa Cruz, Heidelberg, Germany) at $2 \mu \mathrm{g} / \mathrm{mL}$.

\section{Test Reagents and Purification of PV-IgG Fractions}

AK23, a pathogenic Dsg3 monoclonal antibody (mAb) that is derived from a pemphigus mouse model, ${ }^{19}$ was purchased (Biozol, Eching, Germany) and used at a concentration of 75 $\mu \mathrm{g} / \mathrm{mL}$. PV-IgG was collected from a patient with active PV with mucosal and epidermal lesions. The serum was tested by enzyme-linked immunosorbent assay (Euroimmun, Lübeck, Germany) that had both Dsg3 (11,550 U/mL) and Dsg1 (375 U/ $\mathrm{mL}$ ) reactivity. Total IgG was purified as reported previously. ${ }^{20}$

\section{Triton Extraction}

$\mathrm{HaCaT}$ cells were seeded in 24-well plates, transfected with either DP-GFP-control or DP-S2849G-GFP as described above, and incubated for 24 hours with PV-IgG. After a washing step with ice-cold phosphate-buffered saline (PBS), Triton buffer $(0.5 \%$ Triton X-100, $50 \mathrm{mmol} / \mathrm{L}$ MES, 25 $\mathrm{mmol} / \mathrm{L}$ EGTA, and $5 \mathrm{mmol} / \mathrm{L} \mathrm{MgCl}_{2}$ ) was added for $5 \mathrm{mi}-$ nutes on ice. Cells were scraped and lysate was centrifuged at $18,000 \times g$. The pellet corresponds with the cytoskeletal pool, whereas the supernatant is the noncytoskeletal pool. Both fractions were mixed with $3 \times$ Laemmli sample buffer and subjected to Western blot anaylsis.

\section{Electrophoresis and Western Blot Analysis}

The lysates were subjected to gel electrophoresis and blotted according to standard protocols after measurement of protein amount using the bicinchoninic acid method (Thermo Fisher Scientific, Schwerte, Germany). We used DP polyclonal antibody (pAb) (Santa Cruz), Dsg3 pAb (Santa Cruz), $\beta$-actin mAb (Sigma-Aldrich, Taufkirchen, Germany), Dsg2 mAb (Acris, Herford, Germany), glyceraldehyde-3-phosphate dehydrogenase mAb (Santa Cruz), Dsg1 mAb (Progen, Heidelberg, Germany), Dsc1 pAb (Santa Cruz), Dsc2 pAb (Progen), Dsc3 mAb (Progen), E-cadherin mAb (BD Transduction, Heidelberg, Germany), and plakophilin-2 mAb (Progen) as primary antibodies. Either a goat anti-mouse $\mathrm{mAb}$ or a goat anti-rabbit $\mathrm{mAb}$ (Dianova, Hamburg, Germany) was used as the secondary antibody. All antibodies were diluted in 5\% nonfat milk mixed with Tris-buffered saline-Tween. For membrane development, the ECL System (GE Healthcare, München, Germany) was used.

\section{Immunofluorescence}

$\mathrm{HaCaT}$ cells grown to $80 \%$ confluence were transiently transfected with the DP constructs the day after seeding. Forty-eight hours after transfections, the cells were incubated with PV-IgG for 24 hours. Next, the cells were fixed with $2 \%$ formalin (prepared from paraformaldehyde) for 10 minutes, then washed three times with PBS, 5 minutes with Triton X-100 (0.1\%), and further washed three times with PBS. Afterward, the cells were incubated with PBS that contained $10 \%$ bovine serum albumin and 3\% normal goat serum for 30 minutes. A monoclonal Dsg3 antibody (Life Technologies) was used as primary antibody in a 1:100 dilution overnight at $4^{\circ} \mathrm{C}$, followed by several rinses with PBS. Finally, cells were incubated with Cy5-labeled goat anti-mouse (Dianova). After this procedure, a second primary antibody, anti-pan-cytokeratin eFluor $615 \mathrm{mAb}$ (ebioscience, Frankfurt, Germany), was incubated in a 1:100 dilution overnight at $4^{\circ} \mathrm{C}$ in a humid box. Finally, the coverslips were mounted on glass slides with $60 \%$ glycerol in PBS that contained $1.5 \% n$-propyl gallate as an antifading compound. Images of immunostained samples were acquired using a Leica SP5 confocal microscope equipped with a $63 \times$ NA 1.4 PL APO objective (Leica Microsystems, Wetzlar, Germany). 


\section{Dispase-Based Dissociation Assay}

Cells were seeded in 24-well plates, cultured until $80 \%$ confluence, and then transfected transiently with DP-GFP, DP-S2849G-GFP, or GFP. Then, 48 hours after transfection, the cells were incubated with AK23 or PV-IgG for 24 hours. After a short washing step with PBS, dispase II [dissolved in Hanks' balanced salt solution (HBSS); SigmaAldrich] was added for 30 minutes to detach the monolayer from the well bottom. HBSS was added to the dispase solution, and mechanical stress was applied by 10 pipetting cycles using a 1-mL electric pipette (Eppendorf, WesselingBerzdorf, Germany). Finally, the resulting fragments per well were counted under a binocular microscope (Leica). The same procedure was performed for the A431 cells.

For cell dissociation with EGTA, cell monolayers were detached with dispase from well bottoms for 30 minutes. After that, the solution was replaced by HBSS that contained $5 \mathrm{mmol} / \mathrm{L}$ EGTA, and cells were incubated for an additional 4 hours. Finally, cells were placed on an orbital shaker (Stuart, Staffordshire, UK), set to 1000 rpm for 5 minutes.

\section{Immunoprecipitation}

A431 cells were seeded equally in four T75 flasks and cultured until $60 \%$ confluence. Then, medium was exchanged, with either standard medium or $2 \mu \mathrm{g} / \mathrm{mL}$ of doxycycline-containing medium for 24 hours, to induce DPS2849G-GFP. By reaching 95\% confluence, cells were incubated with PV-IgG or PBS for 15 minutes, followed by a short washing step with ice-cold PBS. Next, the cells were incubated in a radioimmunoprecipitation assay buffer $\left(\mathrm{Na}_{2} \mathrm{HPO}_{4}, \mathrm{NaCl}\right.$, Triton X-100, sodium desoxycholat, and SDS) that contained phosphatase and protease inhibitors (Roche, Mannheim, Germany) for 30 minutes on ice before scraping them from the flask bottom. The amount was divided into two parts ( $20 \mu \mathrm{L}$ of each probe was kept for the input lysate), which were incubated overnight with or without phosphorylated serine antibody (no. 9332; Abcam, Cambridge, UK). The next day, the lysates were incubated for 2 hours with $25 \mu \mathrm{L}$ of protein $\mathrm{G}$ beads (Calbiochem, Darmstadt, Germany). Beads were washed several times before denaturing in $30 \mu \mathrm{L}$ of Laemmli buffer for $10 \mathrm{mi}-$ nutes at $95^{\circ} \mathrm{C}$. The whole amount was subjected to gel electrophoresis and incubated with antibodies against DP (Santa Cruz), Dsg3 (Santa Cruz), or plakoglobin (Progen). Membranes were developed as described above.

The same procedure was performed for immunoprecipitating $\mathrm{HaCaT}$ cells but without the addition of doxycycline. The PKC-specific inhibitor Bim-X (Enzo, Lörrach, Germany) was preincubated for 1 hour in a concentration of $1 \mu \mathrm{mol} / \mathrm{L}$.

Analysis of the association of the receptor for activated Ckinase 1 (Rack1) with PKC- $\alpha$ in A431 cells was performed as described above with some modifications. Cells were lyzed in immunoprecipitation buffer that contained Tris$\mathrm{HCl}, \mathrm{NaCl}, \mathrm{SDS}, \mathrm{NP}-40$, EDTA, and $1000 \mu \mathrm{g}$ of lysate and incubated with Rack1 antibody (Santa Cruz). After gel electrophoresis, membranes were probed with antibodies against GFP (Santa Cruz), PKC- $\alpha$ (Abcam), and Rack1.

\section{Image Processing and Statistical Analysis}

Images were processed using Photoshop CS5 (Adobe Systems, San José, CA) and ImageJ version $1.45(\mathrm{NIH}$, Bethesda, MD). For the evaluation of keratin retraction, two distances were measured in cells that expressed DP-GFP or DP-S2849G-GFP: the distance from the nucleus to the peripheral end of the keratin filament web and at the same site the distance from the nucleus to the cell membrane. A ratio of these distances was calculated to delineate keratin retraction. In total, 30 measurements were performed for each condition in three to four independent experiments.

Data were analyzed using Excel (Office 2010; Microsoft, Redmond, WA) and are presented as means \pm SEM. Statistical analysis was performed using Prism version 5 (GraphPad Software, La Jolla, CA). Nongaussian distributed sample values were analyzed using the Kruskal-Wallis test followed by Dunn's post hoc test. For gaussian-distributed values, one-way analysis of variance was performed followed by Bonferroni correction. Statistical significance was assumed when $P<0.05$.

\section{Results}

\section{DP Point Mutation Increases Cell Cohesion after Autoantibody Treatment}

To investigate the effects of the DP-S2849G-GFP, in which the amino acid serine is replaced by glycine, we used two different cell lines. The human keratinocyte cell line HaCaT was transiently transfected with GFP, DP-S2849G-GFP, or DP-GFP (Figure 1A). Transfection efficacy of both DP constructs was similar at approximately $25 \%$ of endogenous DP, and endogenous DP levels were not affected (Supplemental Table S1). In addition, the other major desmosomal proteins tested, namely, Dsg2, Dsg3, Dsc2, Dsc3, plakoglobin, and plakophilin-2, remained unchanged by transfection, as was Ecadherin as major adhesion molecule of adherens junctions (Figure 1B and Supplemental Table S1). As another model, we used the human epidermoid carcinoma cell line $\mathrm{A} 431,{ }^{21}$ which expresses wild-type DP-GFP or DP-S2849G-GFP after the addition of doxycycline for 24 hours. Similar to HaCaT, A431 cells express all major desmosomal proteins with the absence of Dsg1 and Dsc1 (Figure 1C). We did not observe significant changes in the protein amount by induction of the constructs or by doxycycline (Supplemental Table S1). Similarly to HaCaT cells, doxycycline treatment did not alter protein expression (Supplemental Figure S1A and Supplemental Table S2).

Next, we tested the effects of the DP point mutation on cell adhesion by using dispase-based cell dissociation assays in both cell lines. One day after transfection, cells were incubated with AK23, a well-characterized monoclonal antibody derived 

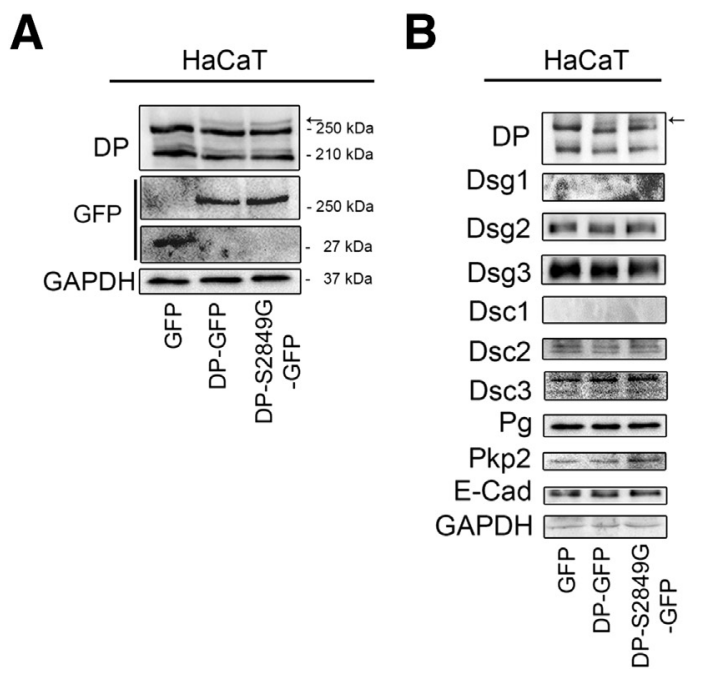

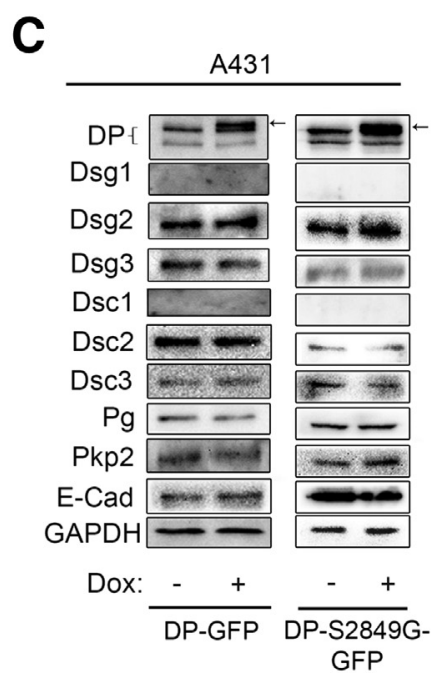

Figure 1 Analysis and characterization of green fluorescent protein (GFP)-tagged desmoplakin (DP) point mutation S2849G (DP-S2849GGFP) in human keratinocyte (HaCaT cells) and epidermoid carcinoma (A431) cells. A: Expression of DP constructs in HaCaT cells. B: Characterization of HaCaT cells transfected with either DP-GFP or DPS2849G-GFP with regard to other cell adhesion molecules. C: Characterization of A431 cells, which express DP-GFP or DP-S2849G-GFP on doxycycline incubation. Arrows denote expression of construct. $n=4$. Dsc, desmocollin; Dsg, desmoglein; E-cad, E-cadherin; GAPDH, glyceraldehyde-3-phosphate dehydrogenase; PKP, plakophilin; Pg, plakoglobin. from a pemphigus mouse model, or with $\mathrm{PV}-\mathrm{IgG}$, an $\mathrm{IgG}$ fraction from a PV patient with autoantibodies against Dsg3 and Dsg1, for an additional 24 hours. Cell monolayers were detached from the well bottom by dispase digestion and subjected to mechanical stress. In GFP- and DP-GFP-transfected $\mathrm{HaCaT}$ cells (Figure 2A), both AK23 and PV-IgG led to loss of cell cohesion after 24 hours, indicated by increased fragmentation of cell sheets compared with controls (Figure 2A). Interestingly, in DP-S2849G-GFP-transfected HaCaT cell monolayers, fragmentation was reduced compared with GFP or DP-GFP-transfected cells. Furthermore, DP-S2849G-GFP reduced AK23- and PV-IgG-induced fragmentation.

DP-GFP-A431 cells not induced with doxycycline and thus not expressing DP-GFP had higher fragment numbers under control conditions compared with $\mathrm{HaCaT}$ cells, which did not change after induction of DP-GFP expression by doxycycline treatment for 24 hours (Figure 2B and
Supplemental Figure S1B). This was similar in HaCaT cells in which doxycycline also had no effect on cell cohesion (Supplemental Figure S1C). Fragment numbers also increased significantly in DP-S2849G-GFP-A431 cells without induction after incubation with AK23 and PV-IgG. However, induction of DP-S2849G-GFP resulted in decreased fragment numbers under control conditions and blocked the effects of AK23 and PV-IgG. Taken together, DP-S2849G-GFP strengthened basal keratinocyte adhesion and reduced autoantibody-induced loss of cell cohesion in both cell lines.

\section{DP-S2849G-GFP Ameliorates Autoantibody-Mediated Keratin Filament Retraction}

A hallmark of PV is the so-called keratin filament retraction, which describes the collapse of the intermediate filament (IF)
A

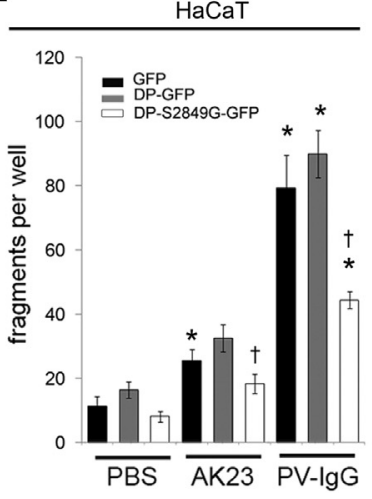

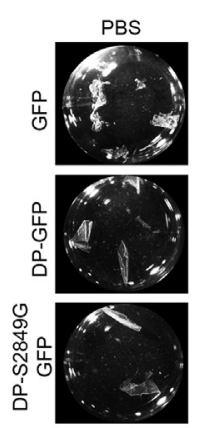

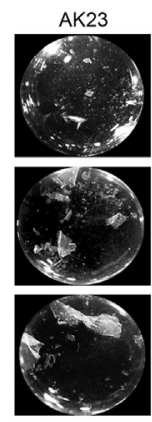

B

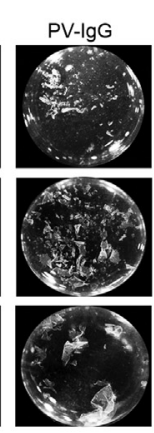

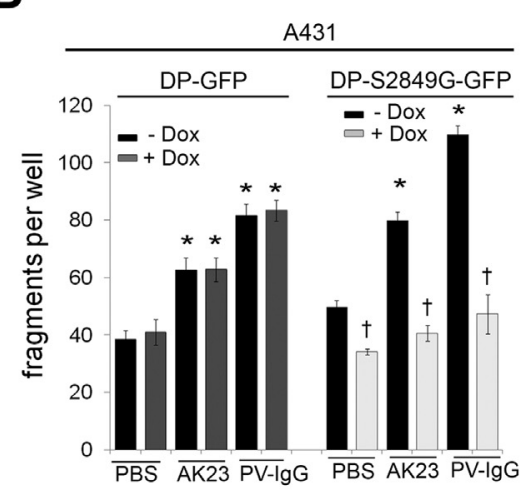

Figure 2 A: Green fluorescent protein (GFP)-tagged desmoplakin (DP) point mutation S2849G (DP-S2849G-GFP) prevents loss of cell cohesion induced by pemphigus vulgaris (PV) autoantibodies. Epidermoid carcinoma AK23 cells and PV-IgG - treated human keratinocyte (HaCaT cell) epithelial sheets transfected with DP-S2849G-GFP display reduced fragmentation compared with wild-type DP-GFP or GFP-transfected cells. B: Representative whole-well views of fragmented monolayer after application of shear stress. Cell dissociation assays with A431 cells. Expression of DP-GFP has no effect, whereas doxycycline-induced (+ Dox) monolayers to express DP-S2849G-GFP resist basal and autoantibody-mediated fragmentation compared with noninduced controls ( - Dox). $n>8$ (A); $n=9$ (B). ${ }^{\star} P<0.05$ versus phosphate-buffered saline GFP $(\mathbf{A})$ or versus -Dox control $(\mathbf{B}) ;{ }^{\dagger} P<0.05$ versus respective DP-GFP condition (A) or versus -Dox (B). PBS, phosphate-buffered saline. 
cytoskeleton and its detachment from desmosomes. ${ }^{1}$ Because DP-S2849G-GFP was found to exhibit increased filament attachment, ${ }^{22}$ we investigated keratin retraction in response to PV-IgG in HaCaT cells (Figure 3A). In cells transfected with wild-type DP-GFP, PV-IgG incubation for 24 hours resulted in IF collapse and detachment from cell borders. This effect was paralleled by pronounced fragmentation of Dsg3 staining. In contrast, in cells transfected with DP-S2849G-GFP, IF distribution was similar to controls and remained unaffected after PV-IgG incubation, whereas it was still detectable in adjacent nontransfected cells. Analysis of keratin retraction (Figure 3B) was achieved as follows: quantification was performed by measuring the distance from the nucleus to the peripheral end of the main mass of the keratin filament web and at the same site the distance from the nucleus to the cell membrane (indicated by residual Dsg3 and the end of singular remaining keratin filaments). A ratio of these distances was calculated to delineate keratin retraction. Concomitantly, fragmentation of Dsg3 staining was also ameliorated by the DP mutant. Thus, DP-S2849G-GFP blocked keratin-filament retraction and fragmentation of Dsg3 staining in response to PV-IgG.

\section{DP-S2849G-GFP Leads to Hyperadhesion and Prevents Desmosomal Dsg3 Depletion within the Desmosome}

It is well established that PV autoantibodies induce depletion of Dsg $3,{ }^{12}$ and this process is pronounced in immature and nonhyperadhesive desmosomes. ${ }^{16,17}$ Similar to a recent study, DP-S2849G-GFP rendered keratinocytes $\mathrm{Ca}^{2+}$ insensitive because fragment numbers were reduced after a 4-hour incubation period with $5 \mathrm{mmol} / \mathrm{L}$ EGTA in $\mathrm{HaCaT}$ cells ( $27.4 \pm 1.7$ for GP versus $20.8 \pm 3.3$ for DP-GFP versus $7.2 \pm 2.7$ DP-S2849G-GFP, Figure 4A). Fragment numbers after EGTA treatment were higher in A431 cells; nevertheless, monolayer fragmentation was reduced by induction of DP-S2849G-GFP but not DP-GFP expression (Figure 4B). Next, we investigated the effect of the DP mutant on Dsg3 depletion in noncytoskeletal (Triton X-100 soluble) and cytoskeletal-attached (Triton X-100 insoluble) protein fractions (Figure 4C). Interestingly, DP-S2849G-GFP-transfected $\mathrm{HaCaT}$ cell monolayers in the cytoskeletal-attached fraction exhibited significantly increased Dsg3 levels under control conditions compared with DP-GFP-transfected cells (Figure 4D). As expected, 24 hours of incubation with PVIgG in DP-GFP-transfected cells led to depletion of Dsg3 in both fractions. However, in cells expressing the DP mutant, Dsg3 depletion was blocked in the cytoskeletonanchored fraction.

\section{DP Is Phosphorylated in Response to PV-IgG Incubation in a PKC-Dependent Manner}

Because the point mutation in DP-S2849G-GFP resides in a putative PKC phosphorylation site ${ }^{7}$ and activation of $\mathrm{PKC}$ is known to participate in $\mathrm{PV}$ - $\operatorname{IgG}-$ mediated cell dissociation, ${ }^{23}$ we investigated whether DP is phosphorylated in response to PV-IgG (Figure 5A). After pull-down of phosphorylated serine in uninduced A431-DP-GFP and A431DP-S2849G-GFP lysates, DP was co-precipitated and the amount of phosphorylated DP was increased after 15 minutes of PV-IgG treatment. After expression of DP-GFP, the fusion protein was also co-precipitated as indicated by a GFP antibody, and the amount of phosphorylated DP after PV-IgG incubation was further enhanced in this cell line. Interestingly, after expression of DP-S2849G-GFP, the amount of phosphorylated DP was reduced compared with uninduced controls and not further increased by PV-IgG treatment. Because no GFP was detectable in the pull-down of the Doxinduced cells, we conclude that the phosphoserine antibody precipitated the native protein but not the mutant. This finding indicates that the mutant indeed is phosphorylation deficient. Similarly, in HaCaT cells, DP was phosphorylated after 15 minutes of PV-IgG treatment, which was blunted by 1 hour pre-incubation with the $\mathrm{PKC}$-specific inhibitor Bim-X (Figure 5B). In contrast, no changes were detectable in
A

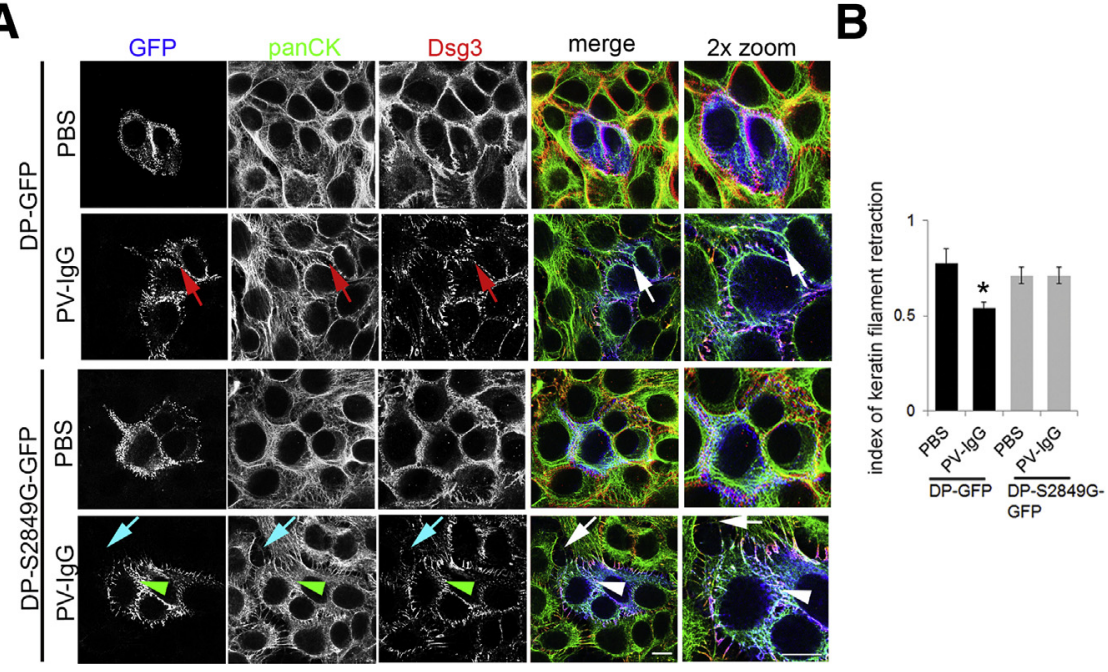

Figure 3 A: Green fluorescent protein (GFP)tagged desmoplakin (DP) point mutation S2849G (DP-S2849G-GFP) blocks autoantibody-mediated keratin retraction. In DP-GFP-transfected human keratinocytes ( $\mathrm{HaCaT}$ cells), keratin retraction and fragmentation of desmoglein (Dsg) 3 staining are evident after pemphigus vulgaris (PV) IgG incubation (red arrows). B: In cells expressing DPS2849G-GFP, keratin filament reorganization and Dsg3 fragmentation is blocked (green arrowheads), whereas these effects are still present in untransfected cells (cyan arrows). Quantification of keratin retraction from four independent experiments. $n=30 .{ }^{*} P<0.05$ versus phosphatebuffered saline (PBS). Scale bar $=10 \mu \mathrm{m}(\mathbf{A})$. 
A

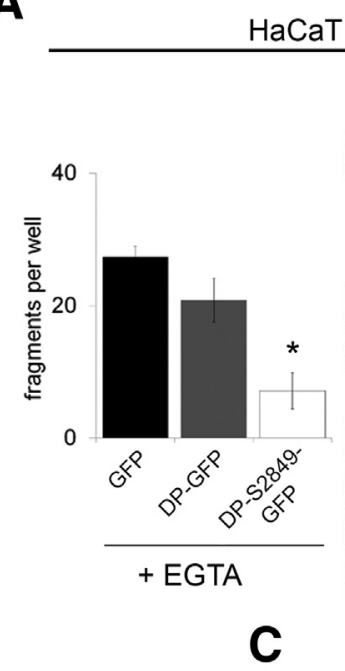

B

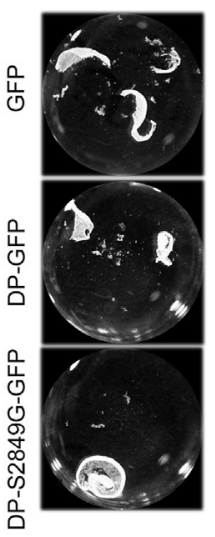

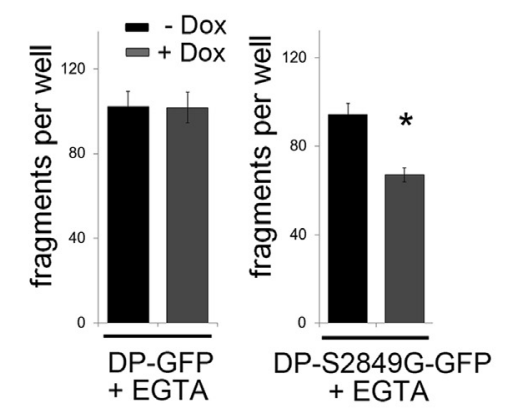

A431

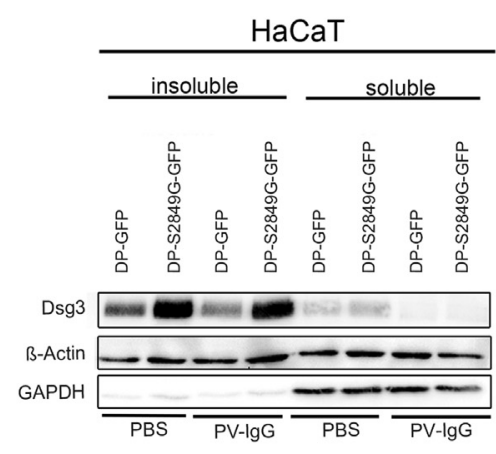

D

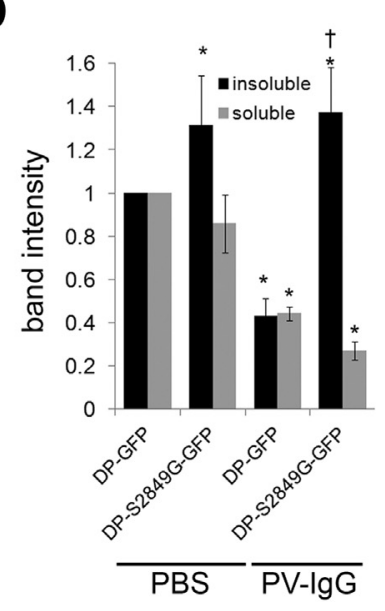

Figure 4 A: Green fluorescent protein (GFP)-tagged desmoplakin (DP) point mutation S2849G (DP-S2849G-GFP) promotes a Ca ${ }^{2+}$-independent state and blocks desmoglein (Dsg) 3 depletion. Cell dissociation assays after treatment with $5 \mathrm{mmol} / \mathrm{L} \mathrm{EGTA}$ for 4 hours reveal Ca ${ }^{2+}$ insensitivity after expression of DP-S2849G-GFP but not DP-GFP in human keratinocytes (HaCaT cells). B: A431 cells are less resistant to EGTA treatment; however, reduced fragmentation is only evident in cells that express DP-S2849G-GFP but not DP-GFP. C: DP-S2849G-GFP increases Dsg3 levels in the Triton X-100 insoluble pool and prevents Dsg3 depletion induced by pemphigus vulgaris (PV) IgG. D: Analysis of Dsg3 band intensity in Triton soluble and insoluble fractions. $n=6(\mathbf{A}$ and $\mathbf{B}) ; n=5$ (C). ${ }^{*} P<0.05$ versus respective PBS DP-GFP condition; ${ }^{\dagger} P<0.05$ versus respective PV-IgG DP-GFP condition.

plakoglobin phosphorylation. These data indicate that PVIgG causes DP phosphorylation at S2849 via PKC.

\section{DP-S2849G-GFP Increases the Association of Rack1 with PKC}

Next, we investigated how DP-S2849G-GFP reduced the amount of phosphorylated endogenous DP-GFP (Figure 5A). Recently, it was suggested that the PKC scaffold Rack1 localizes to keratin filaments and sequesters PKC- $\alpha$, which in turn prevents DP phosphorylation. ${ }^{24}$ Therefore, we speculated that the reduced amount of phosphorylated DP in A431-S2849GGFP lysates (Figure 5A) is a result of increased PKC sequestration. Indeed, the amount of PKC- $\alpha$ associated with Rack1 was increased in A431 cells expressing DP-S2849G-GFP compared with those with DP-GFP expression (Figure 6, A and B). Thus, PKC sequestration by Rack1 may prevent the phosphorylation of endogenous DP in response to PV-IgG.
Finally, because PKC-dependent DP phosphorylation was important for the stability of the desmosome-keratin complex and in a recent study we found that PKC contributes to loss of cell cohesion in pemphigus, ${ }^{25}$ we evaluated whether IF retraction in response to PV-IgG is dependent on PKC. HaCaT cells were treated with PV-IgG for 24 hours, which induced pronounced keratin retraction and Dsg3 fragmentation (Figure 6, C and D). In contrast, simultaneous co-incubation with $\mathrm{Bim}-\mathrm{X}$ prevented keratin retraction in response to $\mathrm{PV}-\mathrm{IgG}$, indicating that $\mathrm{PKC}$ is involved in keratin retraction.

\section{Discussion}

We found that keratinocytes expressing DP-S2849G-GFP were less susceptible to the pathogenic effects of pemphigus autoantibodies on cell adhesion. In addition, expression of DP-S2849G-GFP but not DP-GFP completely blocked 
PV-IgG-mediated cytokeratin retraction, induced formation of $\mathrm{Ca}^{2+}$-independent, hyperadhesive desmosomes, and reduced both desmosomal Dsg3 depletion and DP phosphorylation, two phenomena mediated by PV-IgG via PKC.

\section{Inhibition of PKC-Mediated DP Phosphorylation May Ameliorate Autoantibody-Induced Loss of Cell Cohesion in Pemphigus}

Internalization and depletion of Dsg3 together with keratin retraction are hallmarks of $\mathrm{PV}$. The underlying mechanisms, however, are still incompletely understood. PKC activation in response to PV-IgG has been reported before, although it was unclear which mechanisms are involved in PKCmediated loss of cell cohesion. We found a protective effect of a DP construct with a point mutation in a PKC consensus sequence. Doxycycline-induced expression of this mutant was even sufficient to increase basal cell cohesion, which was not observed in cells with wild-type DP expression or in untransfected HaCaTs treated with doxycycline. Thus, we can exclude that doxycycline was responsible for the increase in cell adhesion. Rather, our data identify PKCdependent DP phosphorylation at $\mathrm{S} 2849 \mathrm{G}$ as an important event in response to pemphigus antibodies. On the basis of the data from this and previous studies, ${ }^{16,17,25}$ both inhibition of PKC and expression of the mutant i) prevented Dsg3 depletion, ii) blocked keratin retraction, and iii) prevented loss of cell cohesion in response to PV-IgG. Because the DPS2849G mutant also regulates hyperadhesion, one may conclude that reversion of the $\mathrm{Ca}^{2+}$-independent phenotype is induced by pemphigus autoantibodies, which in turn destabilizes the desmosome and allows Dsg3 depletion and keratin retraction. Although nothing is known about DP mutations in PV patients, one may speculate that a patient with a mutation in DP-S2849 would experience a milder clinical phenotype. Reduced DP phosphorylation may explain the effectiveness of PKC inhibitors to block blistering in pemphigus mouse models ${ }^{16,26}$ and provide a rationale for more specific treatment of patients with PV.

Another interesting question is whether the sequence of events finally leading to loss of cell adhesion can be concluded from this study (ie, whether keratin retraction is a cause or a consequence of fragmentation of Dsg3 staining, the latter of which was found to reflect Dsg3 depletion). ${ }^{14}$ From the data of this study, we conclude that PKCmediated DP phosphorylation is involved in keratin filament retraction and contributes to loss of cell adhesion. This finding is consistent with our recent study that found that inhibition of PKC reduces PV-IgG-induced loss of cell cohesion. ${ }^{25}$ Figure $6 \mathrm{C}$ shows that under similar conditions in which PKC inhibition blocked keratin retraction, fragmentation of Dsg3 staining is still observed. It is likely that depletion of Dsg3 is upstream of PKC-mediated keratin retraction. However, a recent study found that clustering of Dsg3 immunostaining in patients' skin did not correlate with alterations of desmosome integrity. ${ }^{27}$ Thus, it is also
A
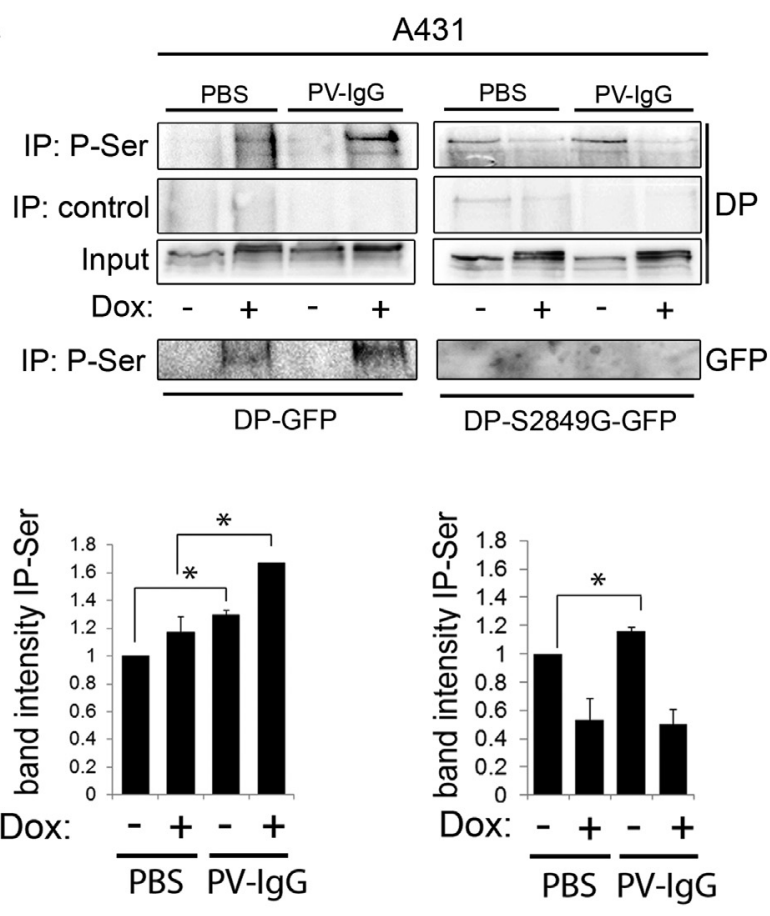

DP-GFP

B

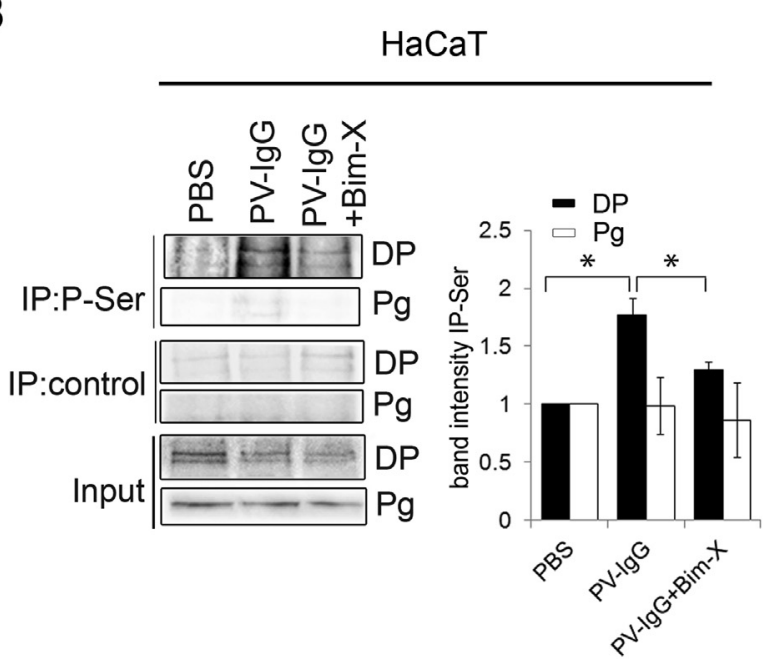

Figure 5 A: Pemphigus vulgaris (PV) IgG induces phosphorylation of desmoplakin (DP). Immunoprecipitation (IP) of phosphorylated serine (P-ser) after 15 minutes of PV-IgG incubation reveals increased DP coimmunoprecipitation in epidermoid carcinoma (A431) uninduced (- Dox) cells compared with uninduced controls. B: A431 cells expressing DP-GFP (+Dox) respond with an increase in DP phosphorylation after PV-IgG treatment. In contrast, DP-S2849G-expressing A431 cells have reduced amounts of DP binding to P-ser. DP is phosphorylated in human keratinocytes ( $\mathrm{HaCaT}$ cells) after PV-IgG, which is blocked by protein kinase C (PKC)-specific inhibitor Bim-X. In contrast, no changes are detectable for plakoglobin (Pg). $n=5(\mathbf{A}) ; n=4$ (B). * $P<0.05$.

possible that the results in Figure $6 \mathrm{C}$ show selective depletion of extradesmosomal Dsg3, whereas desmosomal Dsg3 may be preserved because of inhibition of cytokeratin retraction, which then would indicate that desmosomal Dsg3 
A

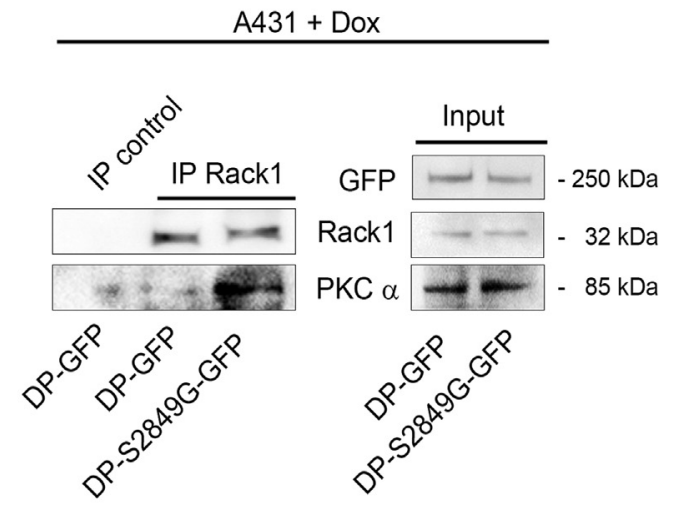

C

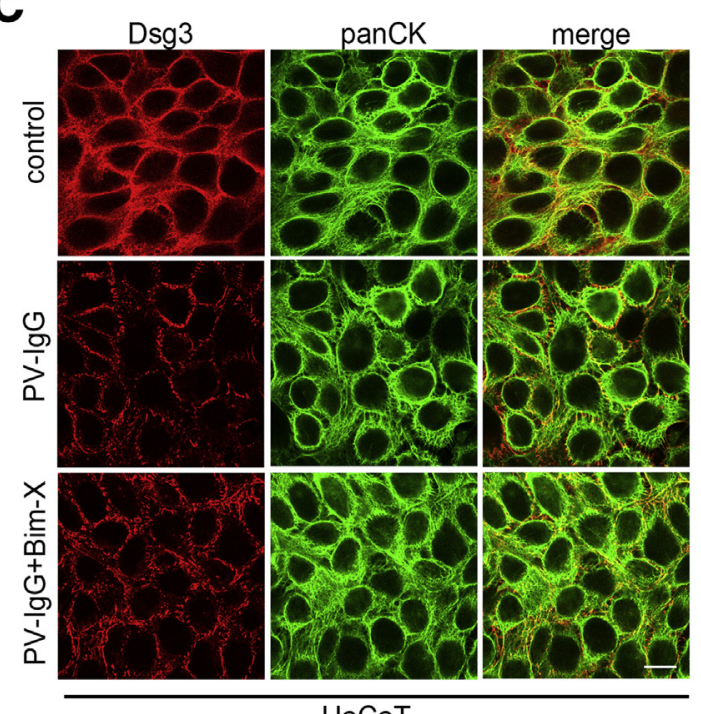

$\mathrm{HaCaT}$

\section{B}

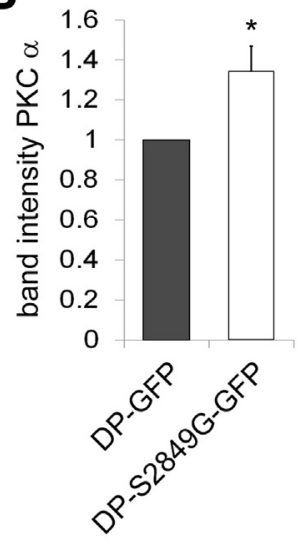

D

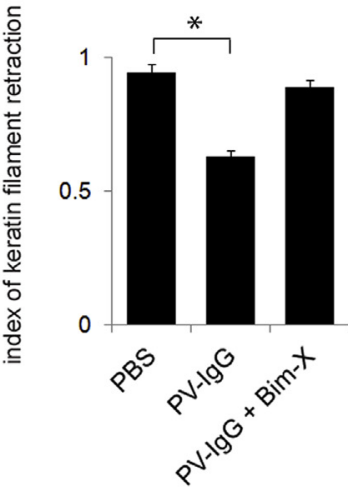

Figure 6 A: Protein kinase C (PKC) sequestration by receptor for activated C-kinase 1 (Rack1) may prevent pemphigoid vulgar (PV) IgG-induced desmoplakin (DP) phosphorylation. Immunoprecipitation of Rack1 reveals increased levels of co-immunoprecipitated PKC $-\alpha$ in epidermoid carcinoma (A431) cells expressing green fluorescent protein (GFP)-tagged DP point mutation S2849G (DP-S2849G-GFP) compared with DP-GFPexpressing A431 cells. B: Analysis of band intensity of five independent experiments. C: Human keratinocytes (HaCaT cells) treated 24 hours with pemphigoid vulgaris (PV) IgG reveal keratin retraction and fragmentation of desmoglein (Dsg)3, which is efficiently blocked after co-incubation with the PKC inhibitor Bim-X. D: Analysis of keratin retraction of three independent experiments ( 54 cells from a total of 6 images per condition). ${ }^{*} P<0.05$. Scale bar $=20 \mu \mathrm{m}(\mathbf{C})$. depletion is a consequence of keratin retraction. Taken together, a clear sequence of these two events in our opinion cannot be determined at present.

\section{Modulation of Keratin Anchorage to the Cytoplasmic Plaque Regulates Desmosomal Hyperadhesion}

DP and the anchorage of keratin filaments to the desmosomal plaque are indispensible for keratinocyte intercellular adhesive strength. $^{28,29}$ In this regard, it is not surprising that the DPS2849G-GFP mutant with ninefold higher keratin filament anchorage $^{22}$ effectively increased cell-cell adhesion. Furthermore, this mutant promoted $\mathrm{Ca}^{2+}$ insensitivity. ${ }^{7}$ Recent data proposed that co-clustering of DP, plakophilin-1, and Dsg3 results in formation of $\mathrm{Ca}^{2+}$-independent and hyperadhesive desmosomes. $^{30}$ Consistent with earlier findings, ${ }^{7}$ our data indicate that replacement of one single amino acid on DP is sufficient to induce a $\mathrm{Ca}^{2+}$-insensitive state, which underlines the importance of phosphorylation of this residue by $\mathrm{PKC}$ for regulation of cell adhesion.
Interestingly, phosphorylation of DP by PKC within the desmosomal plaque is regulated by keratin filaments. We found that DP-S2849G-GFP similar to inhibition of PKC partially prevents the adhesion defect of keratinocytes completely depleted from all keratin filaments. ${ }^{24}$ In this context, keratin filaments sequestered PKC via the adaptor protein Rack1 to prevent DP phosphorylation.

Our data indicate that the association of PKC- $\alpha$ with Rack1 is increased in cells that express DP-S2849G-GFP. It is unclear which mechanisms regulate this enhanced interaction. Nevertheless, on the basis of these data, it may be concluded that the mutant is protective not only because of its insensitivity to phosphorylation but also because it promotes sequestration of PKC to Rack1. Because Rack1 was found to bind to keratins, the increased association of Rack1 with PKC may be related to the enhanced IF anchorage to the mutant DP. Thus, not only do keratin filaments stabilize intercellular cohesion by anchoring the desmosomal plaque to the IF cytoskeleton, which mainly determinates the elastic properties of these cells, ${ }^{31}$ but also keratin filaments 
indirectly regulate desmosomal components (ie, DP phosphorylation via PKC).

\section{Acknowledgments}

We thank Drs. Kathleen Green and Lisa Godsel for providing the DP constructs and A431 cells, Fanny Loschke and Dr. Thomas Magin for helpful discussions, Dr. Ralf Heermann for technical advice, Daniela Kugelmann for discussions, and Angelika Antonius, Marlene Delivuk, Martina Hitzenbichler, Sabine Mühlsimer and Andrea Wehmeyer for skillfull technical support.

\section{Supplemental Data}

Supplemental material for this article can be found at http://dx.doi.org/10.1016/j.ajpath.2014.05.016.

\section{References}

1. Waschke J: The desmosome and pemphigus. Histochem Cell Biol 2008, 130:21-54

2. Delva E, Tucker DK, Kowalczyk AP: The desmosome. Cold Spring Harb Perspect Biol 2009, 1:a002543

3. Yin T, Green KJ: Regulation of desmosome assembly and adhesion. Semin Cell Dev Biol 2004, 15:665-677

4. Yang L, Chen Y, Cui T, Knosel T, Zhang Q, Albring KF, Huber O, Petersen I: Desmoplakin acts as a tumor suppressor by inhibition of the Wnt/beta-catenin signaling pathway in human lung cancer. Carcinogenesis 2012, 33:1863-1870

5. Uzumcu A, Norgett EE, Dindar A, Uyguner O, Nisli K, Kayserili H, Sahin SE, Dupont E, Severs NJ, Leigh IM, Yuksel-Apak M, Kelsell DP, Wollnik B: Loss of desmoplakin isoform I causes early onset cardiomyopathy and heart failure in a Naxos-like syndrome. J Med Genet 2006, 43:e5

6. Jonkman MF, Pasmooij AM, Pasmans SG, van den Berg MP, Ter Horst HJ, Timmer A, Pas HH: Loss of desmoplakin tail causes lethal acantholytic epidermolysis bullosa. Am J Hum Genet 2005, 77:653-660

7. Hobbs RP, Green KJ: Desmoplakin regulates desmosome hyperadhesion. J Invest Dermatol 2012, 132:482-485

8. Garrod DR, Berika MY, Bardsley WF, Holmes D, Tabernero L: Hyper-adhesion in desmosomes: its regulation in wound healing and possible relationship to cadherin crystal structure. J Cell Sci 2005, 118: $5743-5754$

9. Kimura TE, Merritt AJ, Garrod DR: Calcium-independent desmosomes of keratinocytes are hyper-adhesive. J Invest Dermatol 2006, 127:775-781

10. Wallis S, Lloyd S, Wise I, Ireland G, Fleming TP, Garrod D: The alpha isoform of protein kinase $\mathrm{C}$ is involved in signaling the response of desmosomes to wounding in cultured epithelial cells. Mol Biol Cell 2000, 11:1077-1092

11. Amagai M, Stanley JR: Desmoglein as a target in skin disease and beyond. J Invest Dermatol 2012, 132:776-784

12. Yamamoto Y, Aoyama Y, Shu E, Tsunoda K, Amagai M, Kitajima Y: Anti-desmoglein 3 (Dsg3) monoclonal antibodies deplete desmosomes of Dsg3 and differ in their Dsg3-depleting activities related to pathogenicity. J Biol Chem 2007, 282:17866-17876

13. Spindler V, Drenckhahn D, Zillikens D, Waschke J: Pemphigus IgG causes skin splitting in the presence of both desmoglein 1 and desmoglein 3. Am J Pathol 2007, 171:906-916
14. Saito M, Stahley SN, Caughman CY, Mao X, Tucker DK, Payne AS, Amagai M, Kowalczyk AP: Signaling dependent and independent mechanisms in pemphigus vulgaris blister formation. PLoS One 2012, 7:e50696

15. Spindler V, Rotzer V, Dehner C, Kempf B, Gliem M, Radeva M, Hartlieb E, Harms GS, Schmidt E, Waschke J: Peptide-mediated desmoglein 3 crosslinking prevents pemphigus vulgaris autoantibodyinduced skin blistering. J Clin Invest 2013, 123:800-811

16. Spindler V, Endlich A, Hartlieb E, Vielmuth F, Schmidt E, Waschke J: The extent of desmoglein 3 depletion in pemphigus vulgaris is dependent on $\mathrm{Ca}(2+)$-induced differentiation: a role in suprabasal epidermal skin splitting? Am J Pathol 2011, 179:1905-1916

17. Cirillo N, Lanza A, Prime SS: Induction of hyper-adhesion attenuates autoimmune-induced keratinocyte cell-cell detachment and processing of adhesion molecules via mechanisms that involve PKC. Exp Cell Res 2010, 316:580-592

18. Aoyama Y, Owada MK, Kitajima Y: A pathogenic autoantibody, pemphigus vulgaris- $\operatorname{IgG}$, induces phosphorylation of desmoglein 3 and its dissociation from plakoglobin in cultured keratinocytes. Eur J Immunol 1999, 29:2233-2240

19. Tsunoda K, Ota T, Aoki M, Yamada T, Nagai T, Nakagawa T, Koyasu S, Nishikawa T, Amagai M: Induction of pemphigus phenotype by a mouse monoclonal antibody against the amino-terminal adhesive interface of desmoglein 3. J Immunol 2003, 170:2170-2178

20. Waschke J, Bruggeman $\mathrm{P}$, Baumgartner W, Zillikens $\mathrm{D}$, Drenckhahn D: Pemphigus foliaceus IgG causes dissociation of desmoglein 1-containing junctions without blocking desmoglein 1 transinteraction. J Clin Invest 2005, 115:3157-3165

21. Godsel LM, Hsieh SN, Amargo EV, Bass AE, PascoeMcGillicuddy LT, Huen AC, Thorne ME, Gaudry CA, Park JK, Myung K, Goldman RD, Chew T-L, Green KJ: Desmoplakin assembly dynamics in four dimensions: multiple phases differentially regulated by intermediate filaments and actin. J Cell Biology 2005, 171:1045-1059

22. Meng JJ, Bornslaeger EA, Green KJ, Steinert PM, Ip W: Two-hybrid analysis reveals fundamental differences in direct interactions between desmoplakin and cell type-specific intermediate filaments. J Biol Chem 1997, 272:21495-21503

23. Kitajima Y: New insights into desmosome regulation and pemphigus blistering as a desmosome-remodeling disease. Kaohsiung J Med Sci 2013, 29:1-13

24. Kröger C, Loschke F, Schwarz N, Windoffer R, Leube RE, Magin TM: Keratins control intercellular adhesion involving PKC- $\alpha-$ mediated desmoplakin phosphorylation. J Cell Biol 2013, 201:681-692

25. Rötzer V, Breit A, Waschke J, Spindler V: Adducin is required for desmosomal cohesion in keratinocytes. J Biol Chem 2014. epub

26. Sánchez-Carpintero I, España A, Pelacho B, López Moratalla N, Rubenstein DS, Diaz LA, López-Zabalza MJ: In vivo blockade of pemphigus vulgaris acantholysis by inhibition of intracellular signal transduction cascades. Br J Dermatol 2004, 151:565-570

27. van der Wier G, Pas HH, Kramer D, Diercks GFH, Jonkman MF: Smaller desmosomes are seen in the skin of pemphigus patients with anti-desmoglein 1 antibodies but not in patients with anti-desmoglein 3 antibodies. J Invest Dermatol 2014

28. Huen AC, Park JK, Godsel LM, Chen X, Bannon LJ, Amargo EV, Hudson TY, Mongiu AK, Leigh IM, Kelsell DP, Gumbiner BM, Green KJ: Intermediate filament-membrane attachments function synergistically with actin-dependent contacts to regulate intercellular adhesive strength. J Cell Biol 2002, 159:1005-1017

29. Vasioukhin V, Bowers E, Bauer C, Degenstein L, Fuchs E: Desmoplakin is essential in epidermal sheet formation. Nat Cell Biol 2001, 3:1076-1085

30. Tucker DK, Stahley SN, Kowalczyk AP: Plakophilin-1 protects keratinocytes from pemphigus vulgaris $\operatorname{IgG}$ by forming calciumindependent desmosomes. J Invest Dermatol 2014, 134:1033-1043

31. Ramms L, Fabris G, Windoffer R, Schwarz N, Springer R, Zhou C, Lazar J, Stiefel S, Hersch N, Schnakenberg U, Magin TM, Leube RE, Merkel R, Hoffmann B: Keratins as the main component for the mechanical integrity of keratinocytes. Proc Natl Acad Sci U S A 2013, 110:18513-18518 